\title{
Study of the reparative effects of menstrual-derived stem cells on premature ovarian failure in mice
}

\author{
Zhen Wang ${ }^{1}$, Yueling Wang ${ }^{1}$, Ting Yang ${ }^{1}$, Jing $\mathrm{Li}^{2}$ and Xinyuan Yang ${ }^{{ }^{*}}$
}

\begin{abstract}
Background: Young female patients who receive chemotherapy frequently face premature ovarian failure (POF). The therapeutic potential of stem cells in these patients has been explored in stem cells derived from different sources. However, many of these types of stem cells are either difficult to obtain or obtaining them involves invasive procedures. Here, we show that menstrual-derived stem cells (MenSCs) are easy to access and exhibit mesenchymal stem cell-like properties. MenSCs are therefore a novel source of stem cells that can be used for tissue repair. The aim of this study was to explore the reparative capacity and the mechanism underlying the activities of MenSCs.
\end{abstract}

Methods: POF mouse models were established by 7 consecutive days of intraperitoneal injection of cisplatin, and then MenSCs or MenSC-derived conditioned media (CM) were infused via the tail vein. The ovaries were excised after either 7 or 21 days of treatment and the follicles were counted and categorized. Apoptosis of granulosa cells was observed by terminal deoxynucleotidyl transferase mediated dUTP nick end labelling staining. Ovarian function was evaluated by monitoring serum sex hormone levels. Furthermore, MenSC tracking, Q-PCR, and small interfering RNA transfection were used to reveal the inner mechanism of repair.

Results: MenSC transplantation could improve the ovarian microenvironment by reducing apoptosis in granulosa cells and the fibrosis of ovarian interstitium, which contributes to increase the follicular numbers and return sex hormone levels to normal values. Meanwhile, the transplanted MenSCs directively migrate to ovarian interstitium to play a role in repair rather than differentiate to oocytes directly. Additionally, MenSCs and CM derived from these cells exerted protective effects on damaged ovaries partially by secreting FGF2.

Conclusion: MenSCs repair ovarian injury, improve ovarian function, and stimulate regeneration, suggesting that transplantation of MenSCs may provide an effective and novel method for treating POF.

Keywords: Premature ovarian failure, Menstrual-derived stem cells, Paracrine, Fibroblast growth factor 2

\section{Background}

Chemotherapies, which are often used in conjunction with surgery to treat cancer, are unquestionably beneficial therapeutic agents. However, the side effects caused by chemotherapy include damage to the ovaries in female patients, and this effect cannot be ignored because it frequently leads to premature ovarian failure/insufficiency (POF/POI) or menopause as a direct result of its toxic

\footnotetext{
* Correspondence: m18991232845@163.com

'Department of Gynecology and Obstetrics, First Affiliated Hospital, Xi'an

Jiaotong University, Xi'an 710061, People's Republic of China

Full list of author information is available at the end of the article
}

effects on follicles [1-3]. Chemotherapy can even lead to small follicle depletion and a complete loss of oocytes. However, there is currently no clear standard definition for chemotherapy-induced ovarian failure, which is characterized by irreversible amenorrhea in the presence of a negative pregnancy test and follicle-stimulating hormone (FSH) levels higher than $40 \mathrm{mIU} / \mathrm{ml}$ after chemotherapy. A large number of female patients suffer adverse consequences following chemotherapy. These can include hot flushes, cardiovascular or neurological system disease, osteoporosis, and sexual dysfunction. These symptoms are dose dependent and cause a host of serious social and 
psychological problems. Hormone replacement therapy (HRT) has been used to treat POF/POI. However, HRT both increases the risk for recurrence of cancer $[4,5]$ and fails to solve the fundamental problem. In recent years, interest has rapidly grown in studies exploring the therapeutic potential of stem cells because of their potential to differentiate into various specialized cell types. This characteristic has been observed in stem cells derived from a number of different sources, including bone marrow [6-8], amniotic fluid [9], and adipose tissue [10, 11], and all of these cell types have been shown to have therapeutic effects on long-term infertility and ovarian damage. However, many of the suitable cell types currently identified for human use are either difficult to obtain or obtaining them involves invasive procedures.

Menstrual-derived stem cells (MenSCs) display mesenchymal stem cell (MSC)-like properties, including self-renewal, high rates of proliferation, and multi-differentiation capacity [12]. MenSCs can grow and adhere in vitro and have been shown to be positive for MSC markers [13]. Under specific conditions, MenSCs also undergo multipotent differentiation into various functional cell types, including adipocytes [14], myocytes [15], osteocytes [16], cardiomyocytes [17], neurocytes [18, 19], endothelial cells [20], pancreatic cells [21], and hepatocytes [22-25]. In autologous cell repair and regeneration, MenSCs present major advantages over other sources of MSCs, including ease of access and the ability to achieve repeated sampling in a non-invasive manner. Significantly, MenSCs exhibit low immunogenicity and possess immunoregulatory functions which make them safe in xenogenous transplantation [26]. Hence, MenSCs are an ideal cell type for use in treatments for tissue damage. The efficacy of MenSCs has been demonstrated in several clinical trials, including trials evaluating MenSCs as a treatment for myocardial infarction [27], neuron system diseases [28, 29], diabetes mellitus [30,31], and multiple sclerosis [32]. Because of their demonstrated differentiation capacity and plasticity, we hypothesized that MenSCs may also be useful as an alternative treatment for POF/POI.

Therefore, in this study, we injected MenSCs via the tail vein into chemotherapy-induced POF mice and then measured their restorative effect on ovarian function. In addition, we further studied the mechanisms underlying MenSC-mediated repairs.

\section{Methods}

\section{Isolation and culture of cells}

The collection of the samples used for research purposes in this study was approved by the Ethical Committee of The First Affiliated Hospital of Xi'an Jiantong University, and written informed consent was obtained from each donor. Menstrual blood (approximately $10 \mathrm{ml}$ each) was collected from six healthy women (25-30 years old) on the first day of menstruation using a menstrual cup (Green Donna, GuangzhouMeiFanle Rubber Products, China). The samples were then transferred into a $50 \mathrm{ml}$ centrifuge tube containing $10 \mathrm{ml}$ of phosphate-buffered saline (PBS), penicillin $(100 \mathrm{U} / \mathrm{ml})$, streptomycin $(100 \mathrm{mg} / \mathrm{ml}), 0.25 \mathrm{mg} / \mathrm{ml}$ amphotericin $\mathrm{B}$, and $2 \mathrm{mM}$ ethylenediaminetetraacetic acid (EDTA) (Gibco, Grand Island, NY, USA). Menstrual blood mononuclear cells were separated using Ficoll-Paque Plus (GE Healthcare, Amersham, UK) according to the manufacturer's instructions. The cells were suspended in a T25 flask (Corning, New York, USA) containing DMEM/F12 supplemented with $10 \%$ foetal bovine serum (FBS) (Sijiqing, China), streptomycin $(100 \mathrm{mg} / \mathrm{ml})$, and penicillin $(100 \mathrm{U} / \mathrm{ml})$ and then cultured in a humidified incubator at $37{ }^{\circ} \mathrm{C}$ in $5 \%$ $\mathrm{CO}_{2}$. The cell culture medium was changed every 3 days. When the cells reached $90 \%$ confluence, they were detached using $0.25 \%$ trypsin-EDTA and passaged at a ratio of $1: 3$.

\section{MTT assay to evaluate cell proliferation}

Cells at passages P3, P10, and P15 were seeded at $2.0 \times 10^{3}$ cells/well in 96-well plates (Corning) and cultured in DMEM/F12 supplemented with 10\% FBS, streptomycin $(100 \mathrm{mg} / \mathrm{ml})$, and penicillin $(100 \mathrm{U} / \mathrm{ml})$. On each of the following 7 days, MTT $(5 \mathrm{mg} / \mathrm{ml}$; Sigma-Aldrich, St Louis, MO, USA) was added to the cell medium at the same time point, and the cells were then incubated at $37{ }^{\circ} \mathrm{C}$ for an additional $4 \mathrm{~h}$. A $150-\mu$ l volume of dimethylsulfoxide (DMSO; SigmaAldrich) was then added to each well to terminate the reaction, and the blue-violet precipitate was lysed for $15 \mathrm{~min}$ while shaking the solution. Absorbance values were determined at $490 \mathrm{~nm}$ using an ELISA reader (Model 680; Bio-Rad, Hercules, CA, USA). The experiment was performed in triplicate.

\section{Colony-forming assay}

Freshly sorted cells were seeded at a very low seeding density $\left(150\right.$ cells $\left./ \mathrm{cm}^{2}\right)$ in fibronectin-coated $(10 \mu \mathrm{g} / \mathrm{ml})$ $60-\mathrm{mm}$ cell culture dishes and cultured in stromal medium containing DMEM/F12, 10\% FBS, $2 \mathrm{mM}$ glutamine, and $0.5 \mathrm{mg} / \mathrm{ml}$ primocin at $37{ }^{\circ} \mathrm{C}$ in $5 \% \mathrm{CO}_{2}$. The medium was changed every 6-7 days. After 14 days, the cells were fixed in methanol and stained using Giemsa. Only colonies containing 50 or more cells were defined as colony-forming units (CFU). The number of colonies was counted according to this definition.

\section{Flow cytometry analysis}

To analyse surface marker expression using flow cytometry, adherent cells $\left(1 \times 10^{6}\right)$ were harvested in $0.02 \%$ EDTA, washed twice with PBS, and disaggregated into single cell suspensions by pipetting. The cells were 
incubated with $10 \mu \mathrm{l}$ of the following antibodies: fluorescein isothiocyanate (FITC)-conjugated mouse monoclonal antibodies against CD34, CD44, CD45, CD73, and $\mathrm{CD} 90$, and phycoerythrin (PE)-conjugated mouse monoclonal antibodies against CD49, CD133, and CD146 (Biosciences, San Jose, CA, USA) at $4{ }^{\circ} \mathrm{C}$ for $30 \mathrm{~min}$. The cells were then washed twice with PBS, resuspended in $0.4 \mathrm{ml}$ of PBS, and immediately analysed using a FACS Calibur flow cytometer (Becton Dickinson, CA, USA). Cell Quest software was used for the data analysis.

\section{Cytogenetic analysis}

We used cytogenetic analyses to determine the karyotypes of MenSCs at P25. After the MenSCs were treated with Colcemid, they were harvested using centrifugation and washed twice with PBS. Then, $75 \mathrm{mM} \mathrm{KCl}$ was added to the resuspended cells, and the mixture was incubated for $20 \mathrm{~min}$ at $37{ }^{\circ} \mathrm{C}$. To this mixture, we added 7 drops of chilled methanol-acetic acid fixative. The mixture was then centrifuged for $10 \mathrm{~min}$ at $4{ }^{\circ} \mathrm{C}$. The cell suspension was dropped onto a pre-cooled slide and allowed to air dry. The slides were then observed under a microscope.

\section{In-vitro differentiation}

Cells were differentiated into adipogenic and osteogenic lineages to assess their differentiation capacities in vitro.

To achieve adipogenic differentiation, cells were seeded at a density of $1 \times 10^{4}$ cells $/ \mathrm{cm}^{2}$. After the cells reached $80 \%$ confluence, they were incubated in adipogenic differentiation medium for 3 weeks. The cells were then fixed with $4 \%$ paraformaldehyde (Sigma-Aldrich) and stained with Oil Red O (Sigma-Aldrich). The differentiation medium was changed every 3 days. The adipogenic medium contained DMEM/F12, 10\% FBS, $10^{-6} \mathrm{~mol} / \mathrm{L}$ dexamethasone, $0.5 \mathrm{mmol} / \mathrm{L}$ isobutyl methylxanthine, $10 \mu \mathrm{g} / \mathrm{ml}$ insulin, and $200 \mu \mathrm{mol} / \mathrm{L}$ indomethacin (Sigma-Aldrich).

To achieve osteogenic differentiation, the cells were seeded at a density of $2 \times 10^{3}$ cells $/ \mathrm{cm}^{2}$. After $24 \mathrm{~h}$, the medium was replaced with osteogenic differentiation medium, and the cells were induced for 2 weeks. The cells were then fixed, and mineral deposition was visualized using Alizarin Red (Sigma Aldrich). The osteogenic medium contained DMEM/F12, $10 \% \mathrm{FBS}, 10^{-8} \mathrm{~mol} / \mathrm{L}$ dexamethasone, $10 \mathrm{mmol} / \mathrm{L} \beta$-glycerol phosphoric acid, and $50 \mu \mathrm{mol} / \mathrm{L}$ ascorbic acid (Sigma-Aldrich).

At each experimental endpoint, the cell types of the differentiated cells were identified using RT-PCR analysis. The specific primers used for these experiments are presented in Table 1.

\section{Reverse transcription and real-time $\mathrm{qPCR}$}

Total RNA was extracted from the cells using a RNA Fast 200 Kit (Aidlab Biotechnology, China) according to
Table 1 Primer list

\begin{tabular}{lll}
\hline Gene & Forward sequence $\left(5^{\prime} \rightarrow 3^{\prime}\right)$ & Reverse sequence $\left(5^{\prime} \rightarrow 3^{\prime}\right)$ \\
\hline SPARC & TACATCGCCCTGGATGAGTG & CACCTTGCTCCAGGCAGAAC \\
PPARY & GGCCAAGGCTTCATGACAA & AATGGGCTTCACATTCAGCAA \\
Runx2 & TCCACACCATTAGGGACCATC & TGCTAATGCTTCGTGTTCCA \\
FabP4 & AGAGGATGATAAACTGGTGGG & CGAACTTCAGTCCAGGTCAA \\
VEGF & CCTTGCTGCTCTACCTCC & AAATGCTTCTCCGCTCT \\
FGF2 & CTAACCGTTACCTGGCTATG & TTATACTGCCCAGTCGTT \\
IGF-1 & AGGAAGTACATTGAAGAACGCAAGT & CCTGCGGTGGCATGTCA \\
HGF & GATGTCCACGGAAGAGGAGA & GAGTCACCTTCCCTCGATGA \\
G-CSF & GACCCATGGCTGGACCT & ATGGGGAGGGCTTGGCT \\
GAPDH & GCACCGTCAAGGCTGAGAAC & TGGTGAAGACGCCAGTGGA \\
\hline
\end{tabular}

a standard protocol. One microgram of total RNA from each sample was used as the template for the reverse transcription reaction using a RevertAid First Strand cDNA Synthesis Kit (Fermentas, USA). Real-time qPCR was then performed using the cDNA with a SYBR Premix Ex Taq II (Takara, China) and a CFX-96 Realtime PCR Detection System (Bio-Rad, USA). The amplification reaction was performed using 40 cycles of the following conditions: denaturation at $95{ }^{\circ} \mathrm{C}$ for $5 \mathrm{~s}$ and annealing at $60{ }^{\circ} \mathrm{C}$ for $30 \mathrm{~s}$. All gene expression levels were normalized to the level of the internal standard control, Gapdh, and analysed using the $2^{-\Delta \Delta \mathrm{Ct}}$ method. The specific primers used in these experiments are presented in Table 1.

\section{Experimental animals and animal model establishment}

Female C57BL/6 mice aged 7-8 weeks were purchased from Xi'an Jiaotong University Animal Laboratory. The experimental protocol was approved by the Ethical Committee and the Institutional Animal Care and Use Committee of Xi'an Jiaotong University. The body weight of each mouse varied from 16 to $18 \mathrm{~g}$ and was recorded every day. Vaginal smears were obtained daily. The normal oestrous cycle in mice consists of the following four sequential stages: pro-oestrus, oestrus, metoestrus, and dioestrus. These stages were determined based on the presence or absence of leukocytes, cornified epithelium, and nucleated epithelial cells [33, 34]. Additionally, only mice that went through at least two consecutive normal oestrous cycles were included in the experiments.

Mice were intraperitoneally injected with cisplatin (CDDP; $2 \mathrm{mg} / \mathrm{kg}$ ) for 7 consecutive days to create the POF model.

\section{MenSC transplantation}

The mice were randomly divided into the following three groups: control group (normal mice without any treatment, $n=10$ ), POF group (POF mice, $n=20$ ), and 
MenSC-treated group (POF mice that were injected with $200-\mu \mathrm{l}$ cell suspensions containing $2 \times 10^{6}$ MenSCs on days 1 and 3 of the experiment, $n=20$ ). At 7 and 21 days after treatment was begun, the animals were sacrificed, and serum samples and ovaries were collected for subsequent experiments.

\section{Tracking GFP-labelled transplanted MenSCs}

MenSCs were infected with green fluorescence protein (GFP)-expressing lentiviral vectors (Genechem, China). The GFP-expressing MenSCs were cultured in DMEM/ F12 supplemented with 10\% FBS (Sijiqing). Before cell transplantation, GFP expression was verified in the MenSCs using a fluorescence microscope. The cells were trypsinized and washed twice with PBS, and the resulting cell pellet was suspended in PBS to achieve a final density of $5 \times 10^{6}$ cells $/ 100 \mu$ l. Using a Hamilton syringe, a total of $1 \times 10^{7}$ GFP-labelled MenSCs in $200 \mu$ l of PBS were evenly injected into the tail veins of POF mice. At 7 and 21 days after injection, the mice were sacrificed, and frozen sections were made from the ovaries. These were used to investigate the morphologies and locations of GFP-MenSCs under a fluorescence microscope (Olympus, Japan). Nuclei were stained with DAPI.

\section{Ria method of measuring serum hormone}

To analyse ovarian function, collected serum samples were used to measure hormone levels. We measured serum oestradiol (E2) and FSH levels using a $\left[{ }^{125} \mathrm{I}\right]$ Oestradiol Radioimmunoassay Kit (JiuDing, China) and a $\left[{ }^{125} \mathrm{I}\right]$ Human FSH Radioimmunoassay Kit (JiuDing, China) according to a standard protocol.

\section{Ovarian follicle counts and morphologic analysis}

To analyse ovarian morphology, ovaries were collected from the three groups at 7 and 21 days after MenSC transplantation and fixed in $4 \%$ paraformaldehyde for 12-16 h. After the ovaries were fixed, they were dehydrated, paraffin-embedded, serially sectioned at $5 \mu \mathrm{m}$ thick, and mounted on glass microscope slides. Routine haematoxylin and eosin (H\&E) staining was performed for histologic examinations, which were analysed under light microscopy. Follicles were categorized and counted in every fifth section through the ovary. Follicles were classified as follows: a primordial follicle was an oocyte that was surrounded by a single layer of squamous granulosa cells; a primary follicle was an intact, enlarged oocyte with a visible nucleus and one layer of cuboidal granulosa cells; a secondary follicle possessed two or three layers of cuboidal granulosa cells without antral space; early antral follicles contained emerging antral spaces; and pre-ovulatory follicles, the largest of the follicular types, possessed a defined cumulus granulosa cell layer [35].

\section{Apoptosis assay}

To detect apoptosis in the collected ovaries, terminal deoxynucleotidyl transferase mediated dUTP nick end labelling (TUNEL) staining kits (Roche Applied Science, USA) were used according to the manufacturer's instructions. The nuclei were counterstained using DAPI. Images were collected under a fluorescence microscope (Olympus). The percentage of TUNEL-positive cells was determined by counting five random fields from each sample. The results are expressed as the percentages of apoptotic cells in each section.

\section{Conditioned medium administration in vivo}

MenSCs at $80 \%$ confluence were switched to serum-free DMEM/F12 and cultured for an additional $48 \mathrm{~h}$. The culture media were then collected as conditioned media (CM) and concentrated 10 times using ultrafiltration centrifuge tubes (molecular weight cut-off value: $3 \mathrm{kDa}$; Millipore, USA).

Female C57BL/6 mice were sorted into the three following groups: control group (normal animals without any treatment, $n=10$ ), POF group (POF model, approximately $200 \mu \mathrm{l}$ of control DMEM/F12 was injected through the tail vein, $n=20$ ), and CM-treated group (POF model, approximately $200 \mu \mathrm{l}$ of concentrated CM was injected through the tail vein, $n=20$ ). The mice were sacrificed 7 days after treatment. The ovaries of the mice were removed for H\&E staining or TUNEL assays, and serum samples were collected to measure sex hormone levels.

\section{RNA interference}

Fibroblast growth factor 2 (FGF2) small interfering RNAs (siRNAs) and negative transfection control siRNAs (NTC) were purchased from GenePharma (China). Cells were transfected with double-stranded siRNA oligonucleotides specific for FGF2 (sense sequence: 5'-GGGCA GUAUAAACUUGGAUTT-3', anti-sense sequence: 5'AUCCAAGUUUAUACUGCCCTT-3'). MenSCs were incubated with $50 \mathrm{nM}$ siRNA for $6 \mathrm{~h}$. Lipofectamine 2000 reagent (Invitrogen, USA) was used as the transfection reagent. The cells were then switched to fresh DMEM/F12 media. The CM were collected after 48 hours of transfection and used for further experiments. The amount of FGF2 secreted from the MenSCs was measured using enzyme-linked immunosorbent assays (ELISAs).

\section{Enzyme-linked immunosorbent assay}

MenSCs were seeded at $5 \times 10^{5}$ cells/well in six-well plates. Complete medium replacement was performed when the cells reached $80 \%$ confluence. After $48 \mathrm{~h}$, the cells were harvested and spun down in a centrifuge, and the supernatant was collected to measure the amount of FGF2 that was secreted according to the manufacturer's 
protocols (Ameko, China). Optical densities (OD) were measured at $450 \mathrm{~nm}$ using an ELISA plate reader (BioRad, USA).

\section{Statistical analysis}

All analyses were performed using SPSS statistical software (version 15.0; SPSS Inc., Chicago, IL, USA). The data are expressed as the mean \pm SEM from at least three independent experiments. The significance of differences between groups was assessed using one-way analysis of variance (ANOVA), and $P<0.05$ was considered to indicate statistical significance.

\section{Results}

Identification and characterization of MenSCs

The isolated MenSCs exhibited a fibroblast-like spindle morphology (Fig. 1a, b) and maintained stable proliferation capacities. We found that the cells reached the logarithmic phase within 2-6 days, while the stagnate phase began on day 6 (Fig. 1c). To study the ability of MenSCs to self-renew, the cells were dissociated to form single cell suspensions and plated at low density $\left(150\right.$ cells $\left./ \mathrm{cm}^{2}\right)$. Figure 1d shows a typical cloning dish containing colonies of varying sizes after 14 days of culture in vitro. To assess the stability of MenSC a

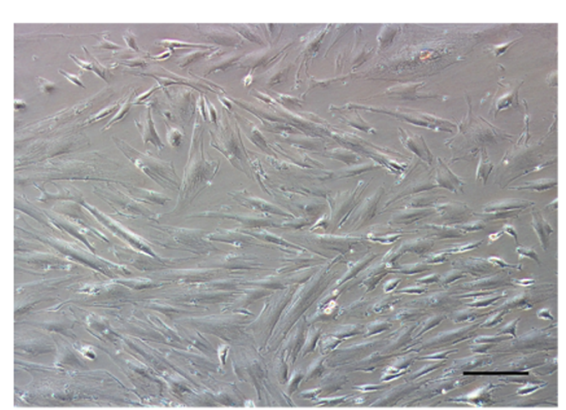

C

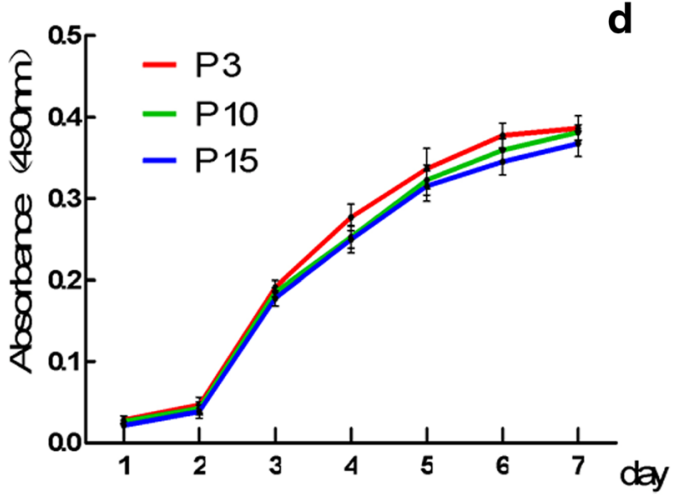

b

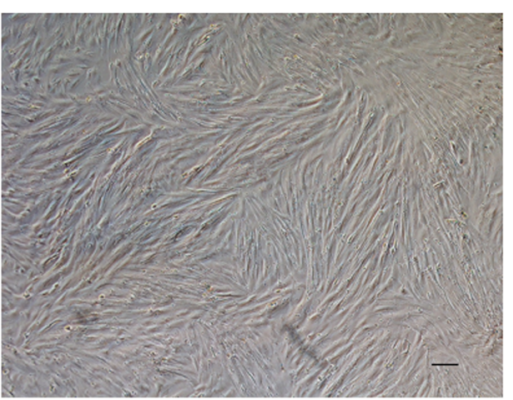

d

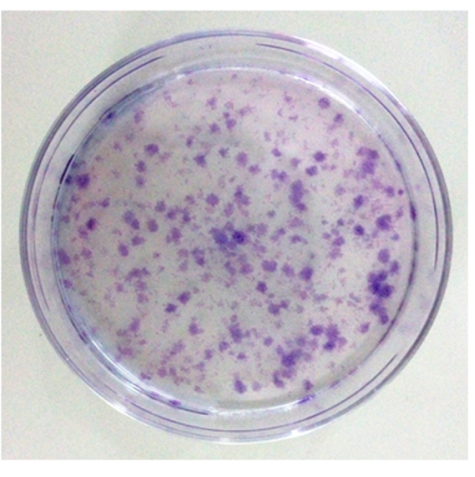

e

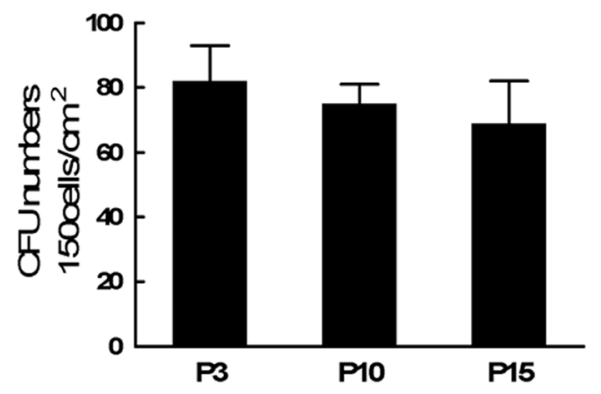

f

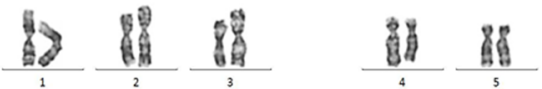

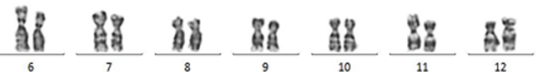

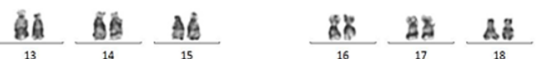

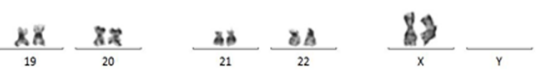

Fig. 1 Proliferation and colony-forming ability of MenSCs. a Morphology of primary MenSCs: passage 0 . Scale bar = $100 \mu$ m. b Morphology of passage cells: passage 3 (P3). Scale bar $=100 \mu \mathrm{m}$. c Growth curves for MenSCs were assessed using MTT assays. d Culture dish displaying the distribution of colonies. e The number of colony-forming units (CFU) was counted during P3, passage 10 (P10), and passage 15 (P15). f Cytogenetic analysis of MenSCs at passage 25 
colony-forming ability, the cells were seeded at different passages (P3, P10, and P15). We found that there was no significant change in colony numbers between early and late passages (Fig. 1e). Additionally, we analysed the karyotypes of MenSCs. Even at passage 25, the cells continued to display a normal karyotype (Fig. 1f).

Flow cytometry was used to identify the surface markers expressed by MenSCs at P3 (Fig. 2a). MenSCs expressed surface markers similar to those expressed by MSCs. These included CD90 (97.23 $\pm 3.2 \%)$, CD44 (99.98 $\pm 1.9 \%)$, CD146 (98.72 $\pm 2.6 \%)$, CD49 (99.91 $\pm 3.3 \%)$, and CD73 $(98.10 \pm 1.9 \%)$. MenSCs did not express haematopoietic cell surface markers, such as CD34 (1.99 $\pm 0.3 \%)$, CD45 (1.93 $\pm 0.1 \%)$, and CD133 (1.87 $\pm 1.9 \%)$.

To determine the multi-differentiation potential of MenSCs, we cultured cells at P3 in osteogenenic or adipogenic differentiation-inducing culture medium. As a result, the cells successfully differentiated into osteoblasts or adipocytes, as shown by histochemical staining (Fig. 2b, c), and expressed the correct tissue-specific markers, as shown by qPCR (Fig. 2d). Mineral deposits were clearly visualized when Alizarin Red staining was used to analyse osteogenic differentiated cells. Furthermore, bone-associated genes, including SPARC and Runx2, were up-regulated in these cells. In adipogenic differentiated cells, lipid droplets were observed to have accumulated when the cells were stained using Oil Red $\mathrm{O}$, and the PPARY and FabP4 genes were up-regulated.

\section{Treatment with MenSCs improves ovarian function in POF mice}

To investigate the effects of MenSC transplantation on ovarian function, we recorded changes across the three groups in body weight at various time points during the study period (Fig. 3a). We consequently observed that body weight was significantly higher in the MenSCtreated group than in the POF group beginning on the 9th day after cell transplantation (Fig. 3b).

We also weighed the ovaries at 7 and 21 days after MenSC transplantation and found that after 21 days the ovaries obtained from MenSC-treated mice weighed significantly more than those obtained from the mice in the POF group (Fig. 3c). In addition, Fig. 3d shows that ovary sizes were different across the three groups after 21 days.

Next, the ovaries in the three groups were collected for pathological analysis. The ovaries in the POF group were more atrophic than the ovaries in the control group, and they exhibited a clear reduction in the number of follicles, especially primordial follicles, during various stages of development (Fig. 3e).

Additionally, we quantified follicle numbers over the course of each treatment. Remarkably, MenSC transplantation substantially increased the number of healthy follicles. After 7 days, there were significantly more primordial follicles $(316 \pm 15.59)$, primary follicles (176 \pm 12.12$)$, secondary follicles $(64 \pm 4.04)$, early antral follicles $(65 \pm 7.51)$, and pre-ovulatory follicles $(38 \pm 2.31)$ in the MenSC-treated group than in the POF group (Fig. $3 \mathrm{f} a$ ). After 21 days, there were significantly more primary follicles $(184 \pm 10.97)$, secondary follicles $(66 \pm 4.62)$, and early antral follicles $(71 \pm 4.62)$ in the MenSC-treated group than in the POF group, and more pre-ovulatory follicles $(36 \pm 2.31)$ and primordial follicles $(342 \pm 9.81)$ than in the POF group but these differences were not statistically significant (Fig. 3fb).

Serum sex hormone levels were significantly different between the MenSC-treated group and the POF group. At 7 and 21 days, the level of E2 was significantly higher, while the level of FSH was lower in the group treated with MenSCs (Fig. 3g, h).

To further explore the protective effects conferred by MenSCs against the chemotherapy-induced loss of follicles, we conducted TUNEL staining at 7 and 21 days after cell infusion and then quantified the number of apoptotic nuclei per total number of nuclei in each section. Interestingly, TUNEL-positive GCs were clearly observed in the POF group, and the results suggested that apoptosis was largely restricted to the GC layer of follicles, in close proximity to the oocytes. In contrast, MenSC transplantation decreased the number of TUNEL-positive cells (Fig. 3i, j), indicating that the MenSCs reduced injury to the ovary by inhibiting apoptosis. Figure 3k shows the percentage of TUNEL-positive cells that were observed in each experimental group.

\section{In-vivo MenSC tracking}

As already shown, MenSCs affect the extent of ovary injury. These cells may be capable of migrating towards to the ovary. We traced the fate of injected MenSCs by injecting mice with GFP-MenSCs. Both ovaries were subsequently harvested from each mouse, and frozen sections were prepared and analysed to detect the number and location of labelled cells. MenSCs transfected with a lentivirus expressing the GFP gene efficiently and stably expressed GFP efficiently in vitro (Fig. 4a). In the mice, we found that GFP-positive cells were located in the interstitium but not in follicles at 7 days after infusion (Fig. 4b), and at 21 days there were no fluorescent signals in the ovaries (Fig. 4c).

\section{Effects of MenSC-derived CM on POF mice in vivo}

As already shown, GFP-positive MenSCs were located in the interstitium, indicating that MenSCs may not differentiate to oocytes directly but that they may improve ovarian function via other mechanisms. Previous studies have demonstrated that MenSCs secrete a variety of 

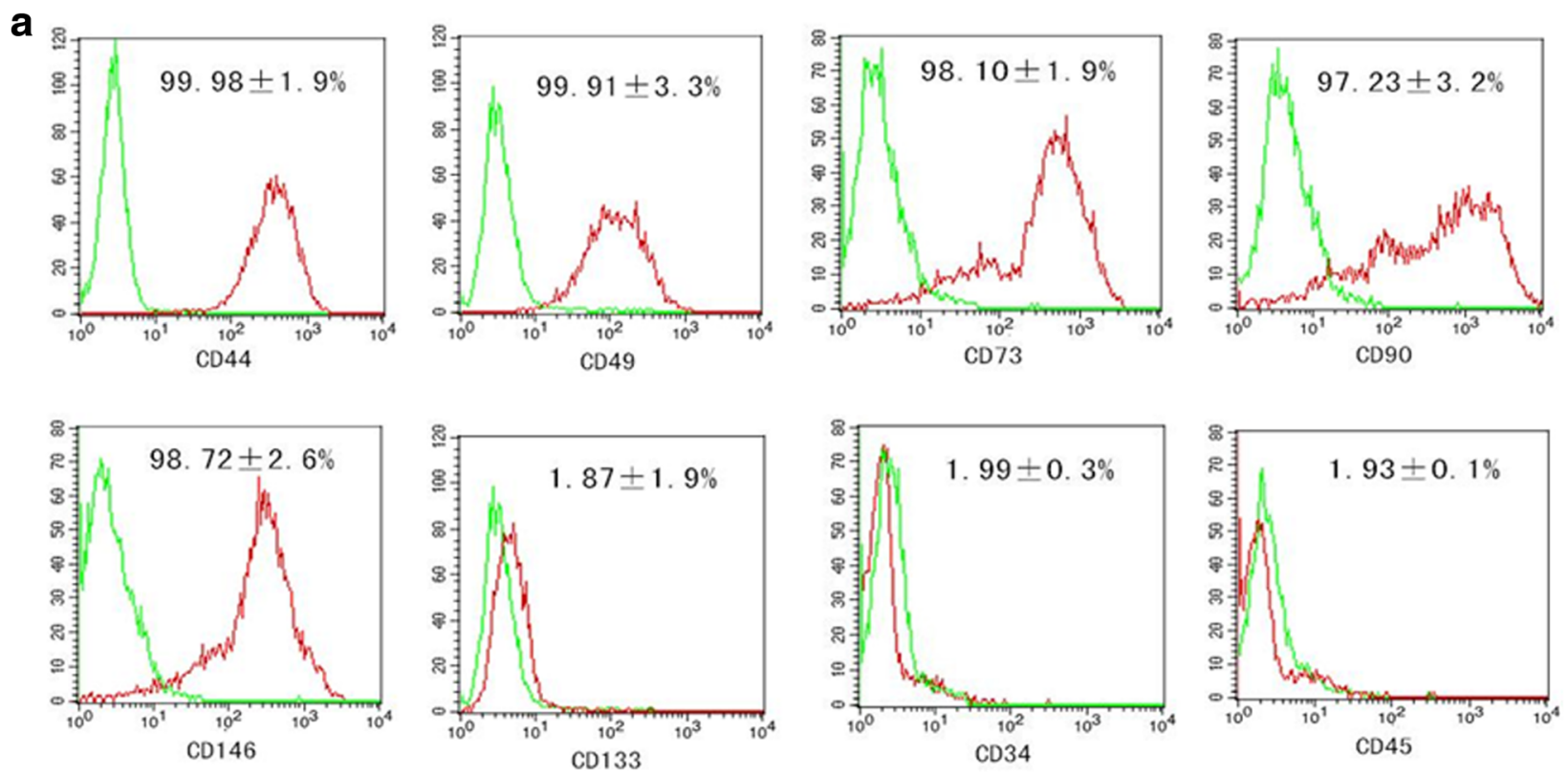

b
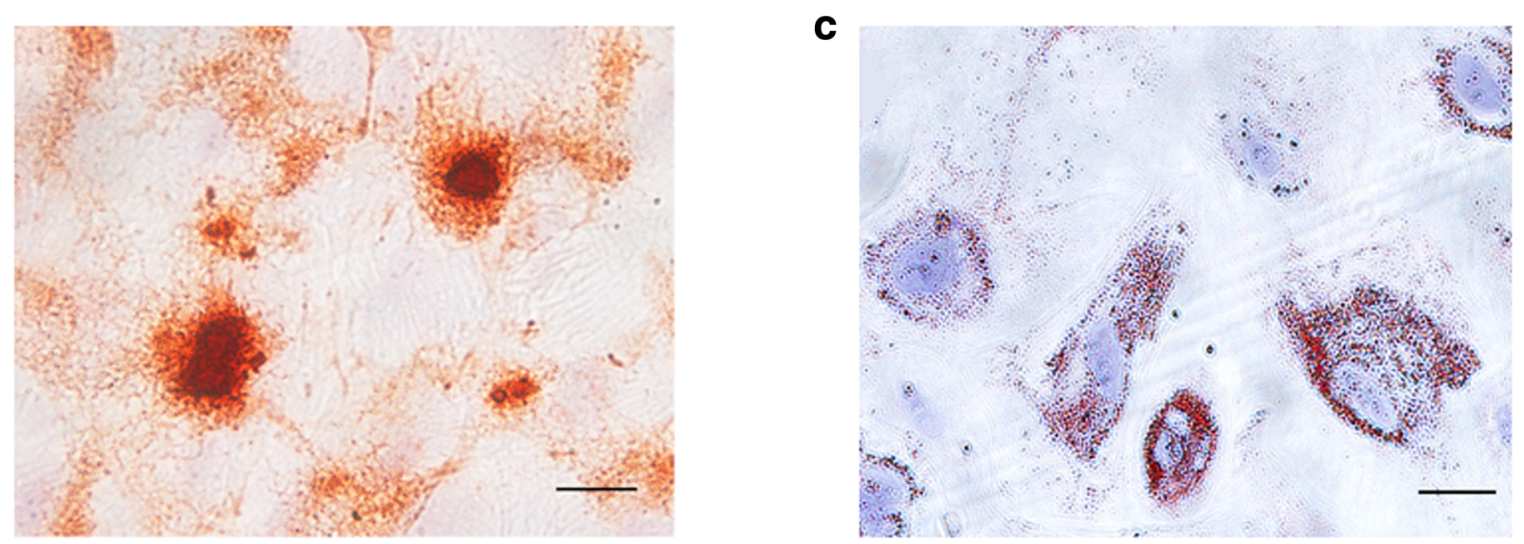

d

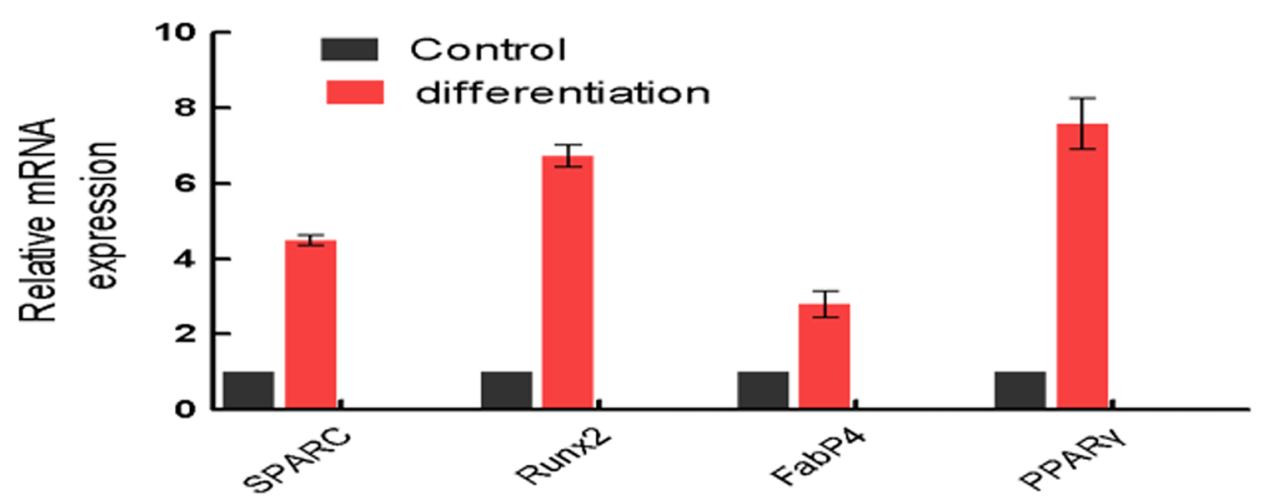

Fig. 2 MenSCs express high levels of mesodermal antigens and display multi-lineage capacities. a Flow cytometry histograms showing the expression level of the indicated cell surface markers on MenSCs. b Multipotent differentiation of MenSCs in vitro: osteogenic differentiation is indicated by Alizarin Red reactivity. Scale bar $=100 \mu \mathrm{m}$. c Multipotent differentiation of MenSCs in vitro: adipogenic differentiation was visualized using Oil Red staining, which reveals lipid droplets. Scale bar $=100 \mu \mathrm{m}$. d Differentiated MenSCs were identified using qPCR analysis to evaluate that expression of osteogenic (SPARC and Runx2) and adipogenic (FabP4 and PPARY) markers. Expression levels of each gene were compared with the levels in undifferentiated MenSCs (data shown as mean \pm SEM) 


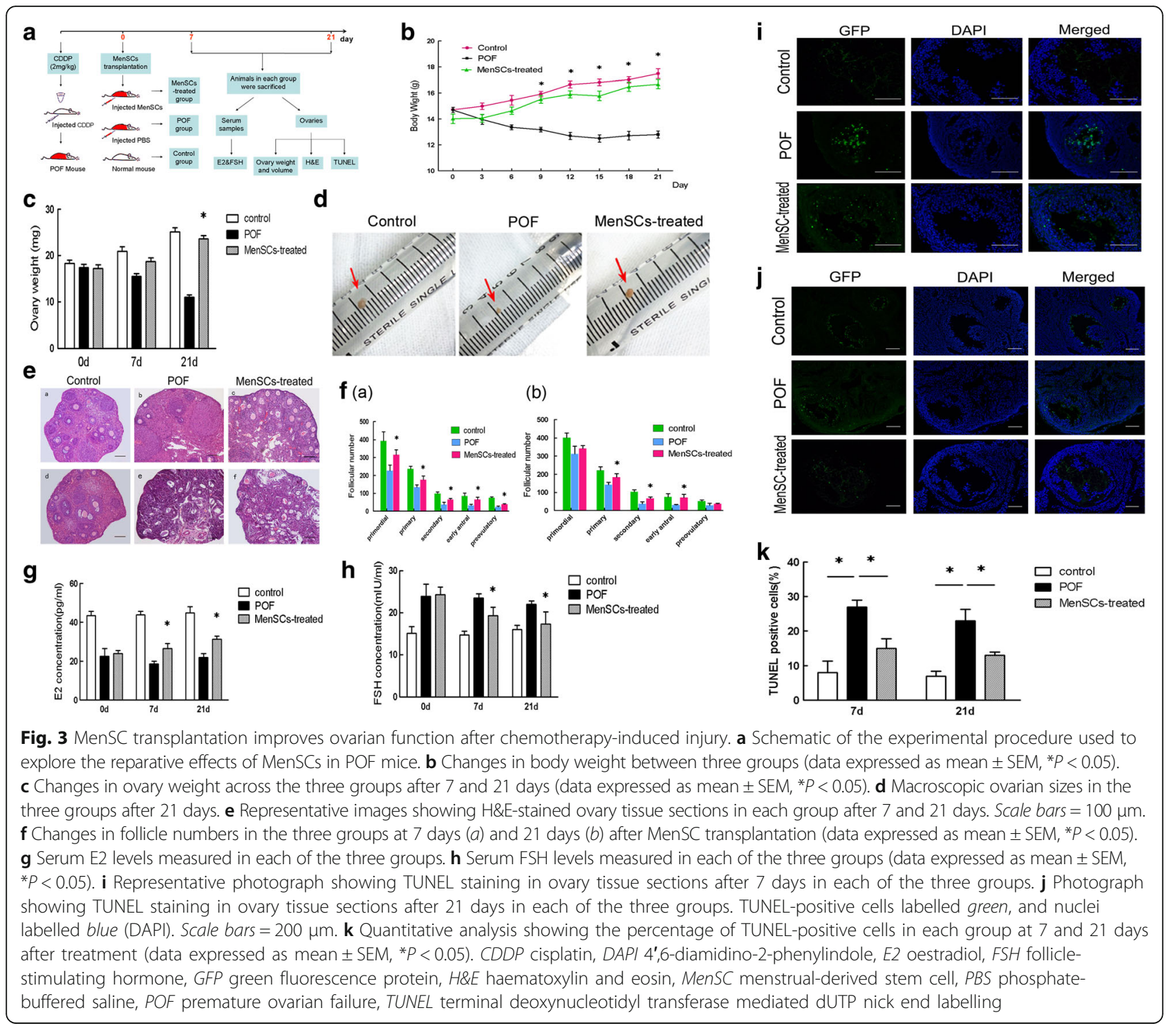

cytokines that could promote the growth and renewal of the human endometrium [36]. To investigate whether MenSCs participate in ovarian recovery via paracrine activity, we collected CM from cultures of MenSCs, and then concentrated and injected them into mice (CMtreated group). The control group was injected with an equal volume of DMEM/F12 instead (Fig. 5a). Remarkably, at 7 days after injection, the levels of E2 were significantly higher and FSH levels were significantly lower in the CM-treated group than in the POF group (Fig. $5 \mathrm{~b}$ and c, respectively). In addition, a study of tissue pathology revealed that ovarian functions were improved and adverse effects were ameliorated after CM administration: the ovarian fibrosis was reduced (Fig. 5d). Moreover, the rate of GC apoptosis was lower in the CMtreated group than in the POF group (Fig. 5e).

\section{FGF2 participates in ovarian injury repairs}

To gain a further insight into the potential ovaryreparative mechanisms underlying the functional benefits observed following treatment with $\mathrm{CM}$ obtained from MenSCs, we evaluated the levels of cytokines and growth factors known to be associated with ovary reparative functions. These included insulin-like growth factor-1 (IGF-1), vascular endothelial growth factor (VEGF), hepatocyte growth factor (HGF), fibroblast growth factor 2 (FGF2), and granulocyte-colony stimulating factor (G-CSF). We determined the level of each markers using qPCR. Interestingly, we found that MenSCs expressed extremely high levels of FGF2 (Fig. 6a).

To further explore the potential ovary-reparative role of FGF2 derived from MenSCs, a siRNA was used to 
a

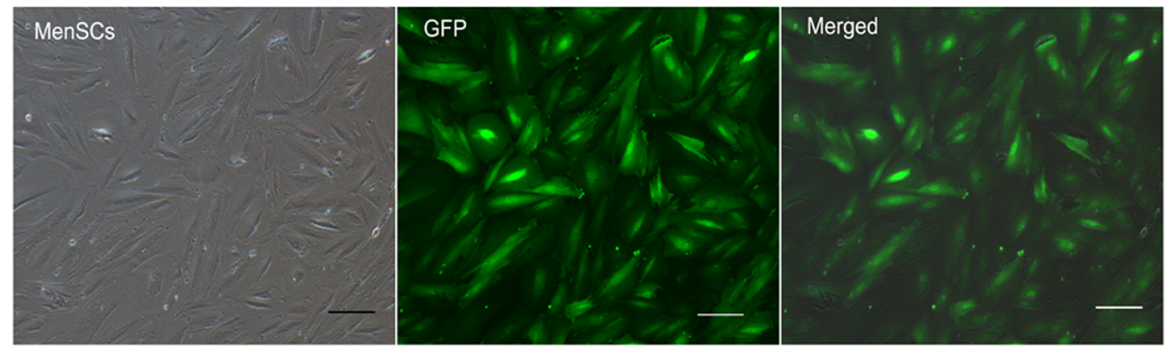

b

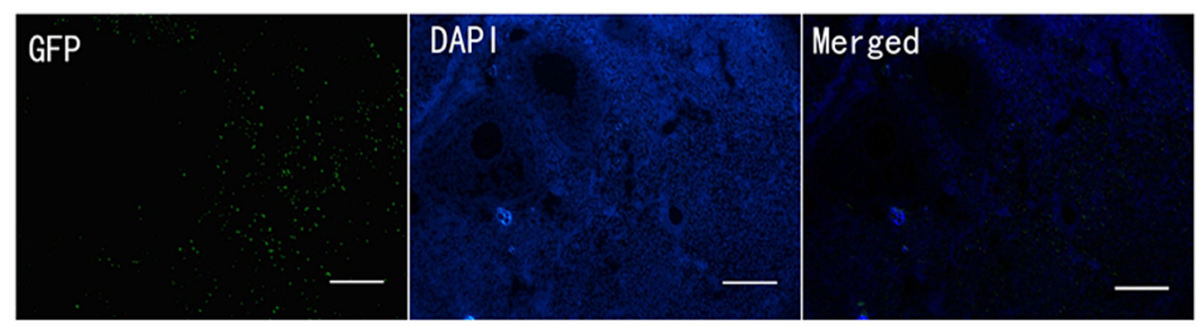

C
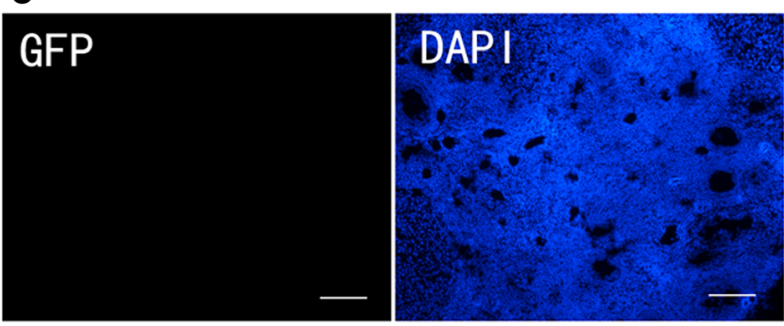

Merged

Fig. 4 In-vivo MenSC tracking. a GFP-MenSCs efficiently expressed GFP in vitro. b Transplanted cells were observed at 7 days after infusion, and almost all of these cells were located in the ovarian interstitium and not in follicles. Scale bars $=100 \mu \mathrm{m}$. c No fluorescent signals in the ovaries at 21 days after cell transplantation. Scale bars $=100 \mu \mathrm{m}$. DAPI 4,6-diamidino-2-phenylindole, GFP green fluorescence protein

disrupt the expression of FGF2. FGF2 was efficiently knocked down at the protein expression level, as shown by ELISA, by the siRNA but not by the NTC (Fig. 6b).

When FGF2 expression was knocked down by the siRNA, the CM obtained from MenSCs no longer significantly increased serum levels of E2 or decreased FSH levels (Fig. 6c, d). Moreover, the reparative effect of the $\mathrm{CM}$ obtained from MenSCs on ovarian injury was also substantially weakened: there were fewer follicles and there was more ovarian interstitial fibrosis in the siRNAtreated group than in the NTC group (Fig. 6e). These data suggest that the therapeutic effect of MenSCs is partially mediated by the secretion of FGF2.

\section{Discussion}

In the present study, we demonstrated that MenSCs isolated from menstrual blood exhibit the properties of MSCs. We explored the potential effects of MenSCs on chemotherapy-induced ovarian damage and found that MenSCs were able to repair ovarian injury successfully.

Previous studies have shown that MSCs can be isolated from many tissues, including skin [37], adipose tissue [10], human amniotic fluid [38], the umbilical cord [39], and the heart [40]. MSCs have become an excellent source of seed cells that can be used for regenerative therapies in a variety of diseases because they have strong self-renewal and multi-differentiation capacities. However, a major drawback of these seed cells is that they were difficult to obtain or the available quantity of them is limited, which restrict their practical applications. Here, we isolated MenSCs from menstrual blood, and we show that they possess the properties of MSCs. Importantly, the most advantageous characteristic of MenSCs, aside from their accessibility and the need for only non-invasive isolation procedures to obtain them, is the possibility that cells can periodically be collected from the same donor. Additionally, as a result of the low immunogenicity and the immunoregulatory function of MenSCs, the allotransplantation of MenSCs has successfully been applied to treatments for multiple sclerosis, myocardial infarction and Parkinson's disease [32, 41, 42]. The allotransplantation of MenSCs in patients with POF can therefore also reasonably be assumed to achieve success. 


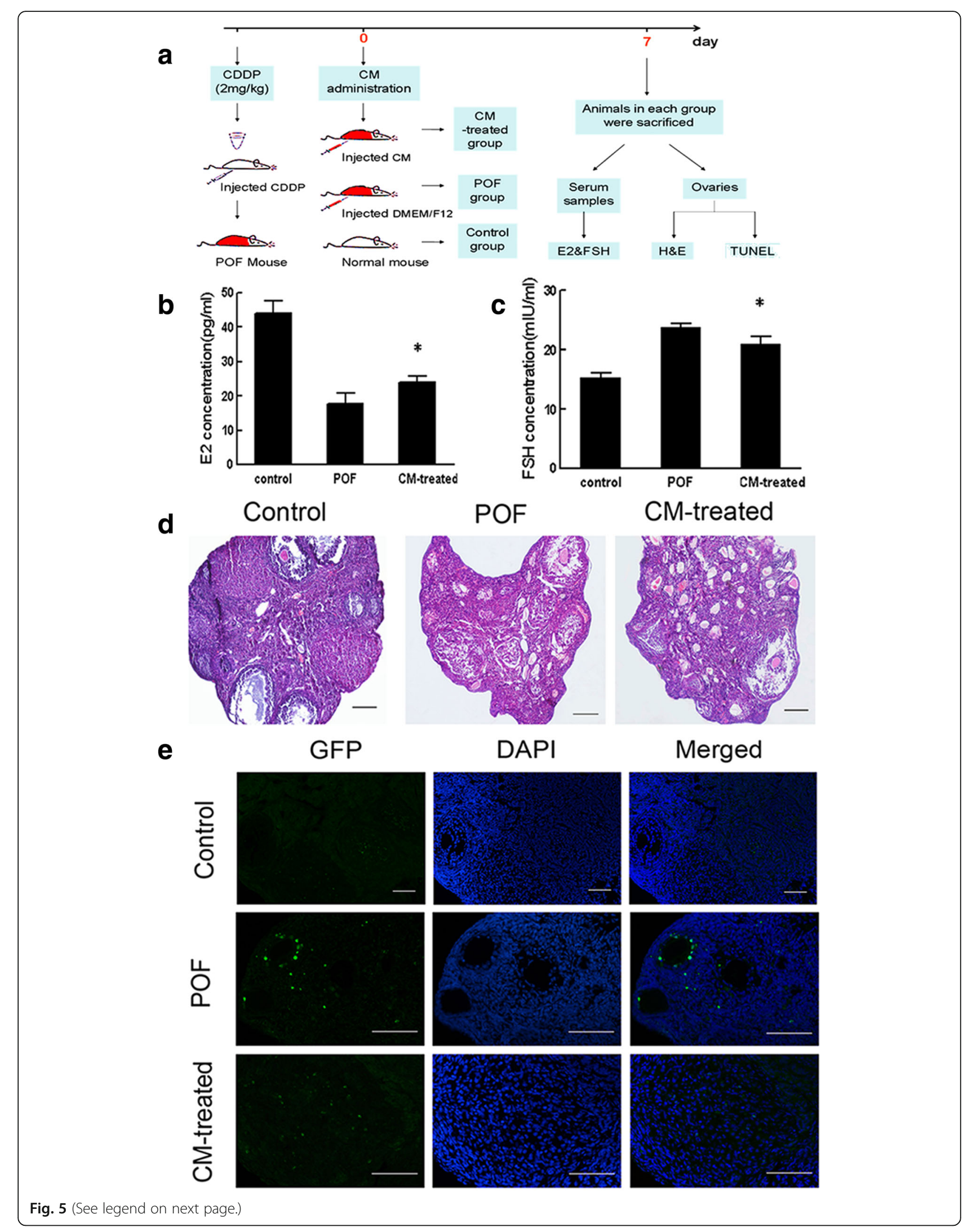


(See figure on previous page.)

Fig. 5 CM obtained from MenSCs improve ovarian function following chemotherapy-induced injury. a Schematic of the experimental procedure used to explore the reparative effects of CM in POF mice. $\mathbf{b}$ Serum E2 levels were measured in each of the three groups after 7 days. c Serum FSH levels were measured in each of the three groups after 7 days (data expressed as mean \pm SEM, ${ }^{*} P<0.05$ ). $\mathbf{d}$ Representative photomicrograph showing the results of H\&E staining in each group at 7 days after injury. Scale bars $=100 \mu \mathrm{m}$. e Apoptosis evaluated using TUNEL staining in each group. Scale bars $=200 \mu \mathrm{m}$. CM conditioned media, CDDP cisplatin, DAPI 4',6-diamidino-2-phenylindole, E2 oestradiol, FSH follicle-stimulating hormone, GFP green fluorescence protein, H\&E haematoxylin and eosin, POF premature ovarian failure, TUNEL terminal deoxynucleotidyl transferase mediated dUTP nick end labelling

Higher therapeutic doses of low-passaged MenSCs with the same genetic background can therefore be obtained from each individual. Moreover, the low immunogenicity of MenSCs suggests that they can be used safely in allotransplantations.
However, as a prominent source of tissues that can be used in cell therapy, MenSCs also possess a broader array of functional properties that might underlie their efficacy for specific therapeutic applications. In this study, we focused on evaluating the cellular and

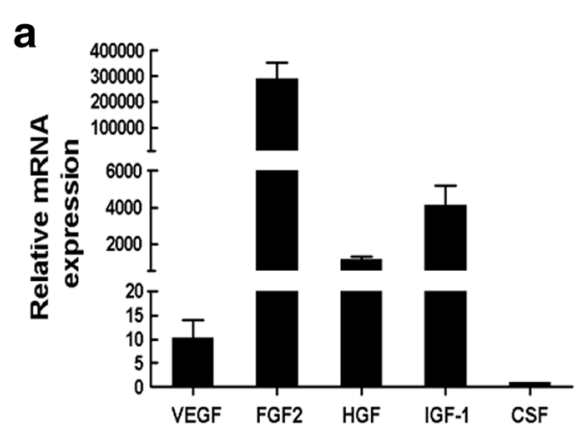

b

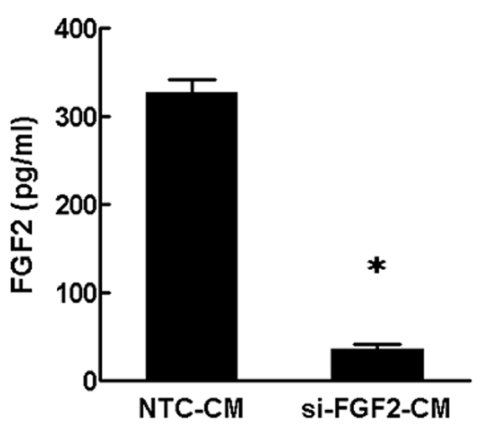

C

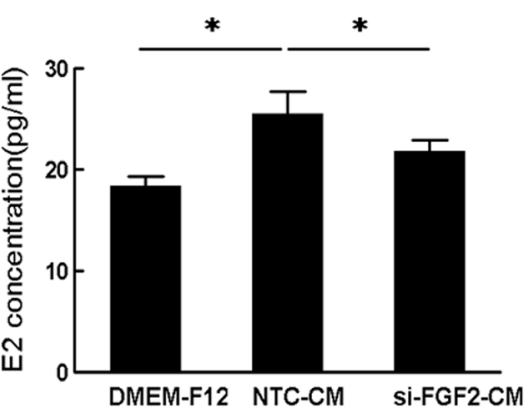

d

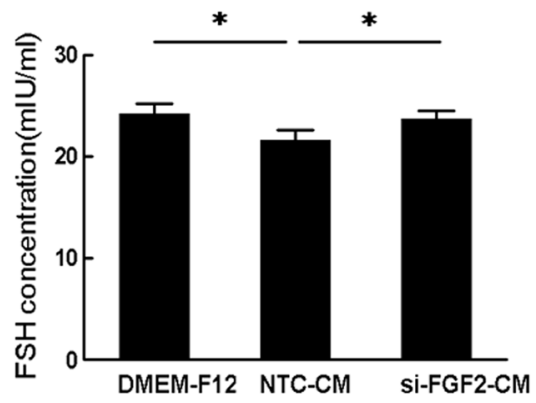

e DMEM/F12 si-FGF2-CM NTC-CM
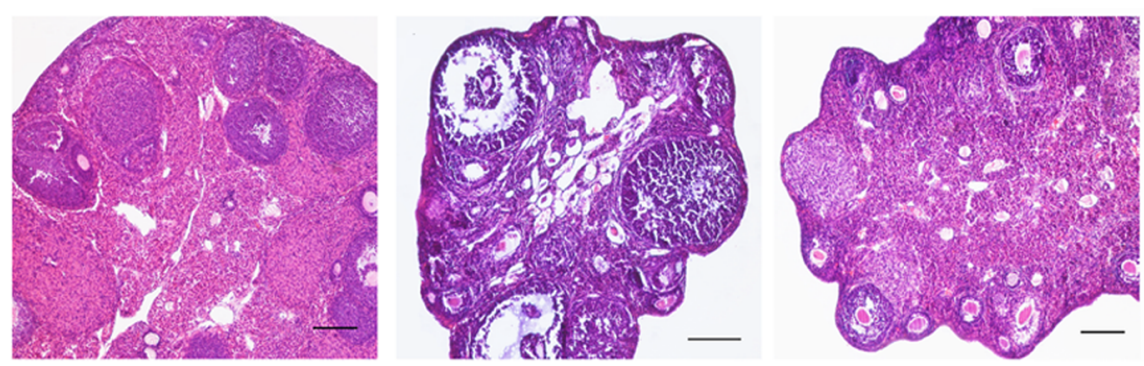

Fig. 6 Restorative effects of MenSCs in POF are mediated via FGF2. a Relative mRNA expression levels of cytokines in MenSCs. b Cells transfected with the FGF2 siRNA expressed significantly lower levels of FGF2, as shown by ELISA (data expressed as mean \pm SEM, ${ }^{*} P<0.05$ ). c Comparison of serum levels of E2 between DMEM/F12-treated, NCT-CM-treated, and si-FGF2-CM-treated mice after 7 days. d Comparison of FSH levels in DMEM/F12-treated, NTC-CMtreated, and si-FGF2-CM-treated mice after 7 days (data expressed as mean $\pm S E M,{ }^{*} P<0.05$ ). e Representative images showing H\&E staining after 7 days. Lesions were more pronounced in the ovaries of si-FGF2-CM-injected mice than in mice treated with NTC-CM. Scale bars: $100 \mu$ m. CM conditioned media, E2 oestradiol, FGF2 fibroblast growth factor 2, FSH follicle-stimulating hormone, NTC negative transfection control siRNAs, IGF-1insulin-like growth factor-1, VEGF vascular endothelial growth factor, HGF hepatocyte growth factor), FGF2fibroblast growth factor 2, CSF colony stimulating factor 
molecular characteristics of MenSCs. The results demonstrate that MenSCs feature characteristics similar to MSCs, including an adherent spindle shape, similar surface marker expression, and multi-lineage differentiation capabilities. We have consequently focused our investigation on examining the reparative effects of MenSCs.

We developed a POF mouse model using cisplatininduced ovarian injury, which has been shown to cause ovarian failure in humans $[1,43]$. Moreover, cisplatin induces apoptosis in GCs [44], which are required for oocyte survival and follicle development [40, 45]. After 7 days, mice that were intraperitoneally injected with cisplatin lost a significant amount of body and ovary weight. Histological analyses indicated that the numbers of follicles in various stages of development were clearly reduced. This was especially true for primordial follicles, which stopped developing and exhibited dysmaturity. Fibrosis was visible in the ovarian stroma, similar to the pathological changes that are observed in clinical cases after treatment with chemotherapy [46]. However, after MenSC transplantation, ovarian function significantly improved: fibrosis was ameliorated, the number of follicles was increased, apoptosis was decreased in GCs, and hormone levels were normalized. Interestingly, we found that while apoptosis was largely restricted to the GC layer of follicles after POF injury, GFP-MenSCs localized to the ovarian stroma rather than follicles, indicating that MenSCs might migrate to the injured areas of the ovary and repair tissues by improving and promoting the regeneration of resident cells. This activity is most probably mediated by paracrine effects that are initiated in response to MenSC-secreted cytokines and growth factors that prevent necrosis and programmed cell death. These cytokines and growth factors may also be instrumental in promoting cell proliferation. We therefore propose that the tissue salvage observed in the MenSCtreated mice included a component of endogenous regeneration as well as ovarian protection, and that these activities were mainly and perhaps exclusively the result of paracrine activity. Previous studies have demonstrated that MSCs express distinct cytokines which enhance cell survival, proliferation, and function and thereby exert a positive effect on repairing tissue damage [47-49]. To research the reparative mechanism underlying the effects of MenSCs, we tested the effects of CM obtained from MenSCs. The results of these experiments confirmed that the MenSC-derived CM played a cytoprotective role and had anti-apoptotic properties. We investigated the expression levels of a variety of cytokines known to be secreted by MenSCs and surprisingly found that these cells produced much higher levels of FGF2 than other cytokines. Among bioactive factors, FGF2 is known to be essential for angiogenesis and for the proliferation and remodelling of endometrial cells, and it has also been shown to play important roles in repairing and regenerative damaged tissues [50-52]. The concentrated $\mathrm{CM}$ that were injected into the mice contained an abundance of FGF2, which effectively repaired ovarian function by decreasing the fibrosis in ovarian interstitium and promoting the growth of follicles. These effects consequently improved the secretion of E2. In spite of the fact that the expression levels of IGF-1 and HGF were slightly higher in MenSCs, when FGF2 expression was knocked using a siRNA the reparative activity of the MenSCs was significantly abrogated. These results further support the notion that the therapeutic effects of MenSCs are likely to be partially mediated by the secretion of FGF2.

\section{Conclusions}

Our research shows that MenSCs can repair ovarian injury, stimulate regeneration, and improve ovarian function. Furthermore, the reparative effects of these cells were mainly based on their paracrine mechanism by secreting FGF2. MenSC transplantation may provide an effective and novel method for treating POF.

\section{Abbreviations \\ CDDP: Cisplatin; CFU: Colony-forming unit; FGF2: Fibroblast growth factor 2; G-CSF: Granulocyte-colony stimulating factor; HGF: Hepatocyte growth factor; HRT: Hormone replacement therapy; IGF-1: Insulin-like growth factor-1; MenSC: Menstrual-derived stem cell; VEGF: vascular endothelial growth factor \\ Acknowledgements \\ Not applicable. \\ Funding \\ This research was supported by the National Natural Science Foundation of China (No. 81571393) and the China Postdoctoral Science Foundation (No.2015 M582676).}

Availability of data and materials

All data generated or analysed during this study are included in this published article.

\section{Authors' contributions}

XYY developed the research question and conceived the study design. ZW conducted most of the experiments. YLW, TY, and JL were involved in some of the experiments. XYY and ZW participated in the statistical analysis and manuscript preparing. All authors read and approved the final manuscript.

\section{Authors' information}

Not applicable.

\section{Competing interests}

The authors declare that they have no competing interests.

\section{Consent for publication}

Not applicable.

\section{Ethics approval and consent to participate}

The experimental protocol about animals was approved by the Ethical Committee and the Institutional Animal Care and Use Committee of Xi'an Jiaotong University.

The collection of the samples used for research purposes in this study was approved by the Ethical Committee of The First Affiliated Hospital of Xi'an Jiantong University, and written informed consent was obtained from each donor. 


\section{Author details}

'Department of Gynecology and Obstetrics, First Affiliated Hospital, Xi'an Jiaotong University, Xi'an 710061, People's Republic of China. ${ }^{2}$ Center for Translational Medicine, First Affiliated Hospital of Xi'an Jiaotong University, Xi'an 710061, People's Republic of China.

Received: 12 August 2016 Revised: 1 November 2016 Accepted: 15 December 2016 Published online: 23 January 2017

\section{References}

1. Nozaki Y, Furubo E, Matsuno T, Fukui R, Kizawa K, Kozaki T, Sanzen T. Collaborative work on evaluation of ovarian toxicity. 6) Two- or four-week repeated-dose studies and fertility study of cisplatin in female rats. J Toxicol Sci. 2009;34 Suppl 1:SP73-81.

2. Kalich-Philosoph L, Roness $\mathrm{H}$, Carmely A, Fishel-Bartal M, Ligumsky $H_{\text {, }}$ Paglin S, Wolf I, Kanety H, Sredni B, Meirow D. Cyclophosphamide triggers follicle activation and "burnout"; AS101 prevents follicle loss and preserves fertility. Sci Transl Med. 2013;5:185ra62.

3. Shirota M, Soda S, Katoh C, Asai S, Sato M, Ohta R, Watanabe G, Taya K, Shirota K. Effects of reduction of the number of primordial follicles on follicular development to achieve puberty in female rats. Reproduction. 2003:125:85-94.

4. Beral V, Banks E, Reeves G. Evidence from randomised trials on the longterm effects of hormone replacement therapy. Lancet. 2002;360:942-4.

5. Miller J, Chan BK, Nelson HD. Postmenopausal estrogen replacement and risk for venous thromboembolism: a systematic review and meta-analysis for the U.S. Preventive Services Task Force. Ann Intern Med. 2002;136:680-90,

6. Johnson J, Bagley J, Skaznik-Wikiel M, Lee HJ, Adams GB, Niikura Y, Tschudy KS, Tilly JC, Cortes ML, Forkert R, Spitzer T, lacomini J, Scadden DT, Tilly JL. Oocyte generation in adult mammalian ovaries by putative germ cells in bone marrow and peripheral blood. Cell. 2005;122:303-15.

7. Lee HJ, Selesniemi K, Niikura Y, Niikura T, Klein R, Dombkowski DM, Tilly JL. Bone marrow transplantation generates immature oocytes and rescues long-term fertility in a preclinical mouse model of chemotherapy-induced premature ovarian failure. J Clin Oncol. 2007;25:3198-204.

8. Santiquet N, Vallieres L, Pothier F, Sirard MA, Robert C, Richard F. Transplanted bone marrow cells do not provide new oocytes but rescue fertility in female mice following treatment with chemotherapeutic agents. Cell Reprogram. 2012;14:123-9.

9. Lai D, Wang F, Chen Y, Wang L, Wang Y, Cheng W. Human amniotic fluid stem cells have a potential to recover ovarian function in mice with chemotherapy-induced sterility. BMC Dev Biol. 2013;13:34.

10. Sun M, Wang S, Li Y, Yu L, Gu F, Wang C, Yao Y. Adipose-derived stem cells improved mouse ovary function after chemotherapy-induced ovary failure. Stem Cell Res Ther. 2013;4:80.

11. Takehara Y, Yabuuchi A, Ezoe K, Kuroda T, Yamadera R, Sano C, Murata N, Aida T, Nakama K, Aono F, Aoyama N, Kato K, Kato O. The restorative effects of adipose-derived mesenchymal stem cells on damaged ovarian function. Lab Invest. 2013;93:181-93.

12. Rossignoli F, Caselli A, Grisendi G, Piccinno S, Burns JS, Murgia A, Veronesi E, Loschi P, Masini C, Conte P, Paolucci P, Horwiz EM, Dominici M. Isolation, characterization, and transduction of endometrial decidual tissue multipotent mesenchymal stromal/stem cells from menstrual blood. Biomed Res Int. 2013;2013:901821.

13. Schwab KE, Gargett CE. Co-expression of two perivascular cell markers isolates mesenchymal stem-like cells from human endometrium. Hum Reprod. 2007;22:2903-11.

14. Khanmohammadi M, Khanjani S, Edalatkhah H, Zarnani AH, Heidari-Vala H, Soleimani M, Alimoghaddam K, Kazemnejad S. Modified protocol for improvement of differentiation potential of menstrual blood-derived stem cells into adipogenic lineage. Cell Prolif. 2014;47:615-23.

15. Fayazi M, Salehnia M, Ziaei S. Differentiation of human CD146-positive endometrial stem cells to adipogenic-, osteogenic-, neural progenitor-, and glial-like cells. In Vitro Cell Dev Biol Anim. 2015;51:408-14.

16. Gargett CE, Schwab KE, Zillwood RM, Nguyen HP, Wu D. Isolation and culture of epithelial progenitors and mesenchymal stem cells from human endometrium. Biol Reprod. 2009;80:1136-45

17. Hida N, Nishiyama N, Miyoshi S, Kira S, Segawa K, Uyama T, Mori T, Miyado K, Ikegami Y, Cui C, Kiyono T, Kyo S, Shimizu T, Okano T, Sakamoto M, Ogawa S, Umezawa A. Novel cardiac precursor-like cells from human menstrual bloodderived mesenchymal cells. Stem Cells. 2008;26:1695-704.
18. Shirian S, Ebrahimi-Barough S, Saberi H, Norouzi-Javidan A, Mousavi SM, Derakhshan MA, Arjmand B, Ai J. Comparison of capability of human bone marrow mesenchymal stem cells and endometrial stem cells to differentiate into motor neurons on electrospun poly(epsilon-caprolactone) scaffold. Mol Neurobiol. 2016;53:5278-87.

19. Asmani MN, Ai J, Amoabediny G, Noroozi A, Azami M, Ebrahimi-Barough S, Navaei-Nigjeh M, Ai A, Jafarabadi M. Three-dimensional culture of differentiated endometrial stromal cells to oligodendrocyte progenitor cells (OPCs) in fibrin hydrogel. Cell Biol Int. 2013;37:1340-9.

20. Shamosi A, Mehrabani D, Azami M, Ebrahimi-Barough S, Siavashi V, Ghanbari H, Sharifi E, Roozafzoon R, Ai J. Differentiation of human endometrial stem cells into endothelial-like cells on gelatin/chitosan/bioglass nanofibrous scaffolds. Artif Cells Nanomed Biotechnol. 2017:45:163-173.

21. Niknamasl A, Ostad SN, Soleimani M, Azami M, Salmani MK, Lotfibakhshaiesh N, Ebrahimi-Barough S, Karimi R, Roozafzoon R, Ai J. A new approach for pancreatic tissue engineering: human endometrial stem cells encapsulated in fibrin gel can differentiate to pancreatic islet beta-cell. Cell Biol Int. 2014;38:1174-82

22. Meng $X$, Ichim TE, Zhong J, Rogers A, Yin Z, Jackson J, Wang H, Ge W, Bogin V, Chan KW, Thebaud B, Riordan NH. Endometrial regenerative cells: a novel stem cell population. J Transl Med. 2007;5:57.

23. Patel AN, Park E, Kuzman M, Benetti F, Silva FJ, Allickson JG. Multipotent menstrual blood stromal stem cells: isolation, characterization, and differentiation. Cell Transplant. 2008;17:303-11.

24. Wu X, Luo Y, Chen J, Pan R, Xiang B, Du X, Xiang L, Shao J, Xiang C. Transplantation of human menstrual blood progenitor cells improves hyperglycemia by promoting endogenous progenitor differentiation in type 1 diabetic mice. Stem Cells Dev. 2014;23:1245-57.

25. Yang $X Y$, Wang $W$, Li X. In vitro hepatic differentiation of human endometrial stromal stem cells. In Vitro Cell Dev Biol Anim. 2014;50:162-70.

26. Xu Y, Zhu H, Zhao D, Tan J. Endometrial stem cells: clinical application and pathological roles. Int J Clin Exp Med. 2015;8:22039-44.

27. Jiang Z, Hu X, Yu H, Xu Y, Wang L, Chen H, Chen H, Wu R, Zhang Z, Xiang C, Webster KA, Wang JA. Human endometrial stem cells confer enhanced myocardial salvage and regeneration by paracrine mechanisms. J Cell Mol Med. 2013:17:1247-60.

28. Wolff EF, Mutlu L, Massasa EE, Elsworth JD, Eugene Redmond Jr D, Taylor HS. Endometrial stem cell transplantation in MPTP-exposed primates: an alternative cell source for treatment of Parkinson's disease. J Cell Mol Med. 2015;19:249-56.

29. Wolff EF, Gao XB, Yao KV, Andrews ZB, Du H, Elsworth JD, Taylor HS Endometrial stem cell transplantation restores dopamine production in a Parkinson's disease model. J Cell Mol Med. 2011;15:747-55.

30. Santamaria X, Massasa EE, Feng Y, Wolff E, Taylor HS. Derivation of insulin producing cells from human endometrial stromal stem cells and use in the treatment of murine diabetes. Mol Ther. 2011;19:2065-71.

31. Li HY, Chen YJ, Chen SJ, Kao CL, Tseng LM, Lo WL, Chang CM, Yang DM, Ku HH, Twu NF, Liao CY, Chiou SH, Chang YL. Induction of insulin-producing cells derived from endometrial mesenchymal stem-like cells. J Pharmacol Exp Ther. 2010;335:817-29

32. Zhong $Z$, Patel AN, Ichim TE, Riordan NH, Wang H, Min WP, Woods EJ, Reid M, Mansilla E, Marin GH, Drago H, Murphy MP, Minev B. Feasibility investigation of allogeneic endometrial regenerative cells. J Transl Med. $2009 ; 7: 15$

33. Felicio LS, Nelson JF, Finch CE. Longitudinal studies of estrous cyclicity in aging C57BL/6 J mice: II. Cessation of cyclicity and the duration of persistent vaginal cornification. Biol Reprod. 1984;31:446-53.

34. Byers SL, Wiles MV, Dunn SL, Taft RA. Mouse estrous cycle identification tool and images. PLoS One. 2012;7:e35538.

35. Myers M, Britt KL, Wreford NG, Ebling FJ, Kerr JB. Methods for quantifying follicular numbers within the mouse ovary. Reproduction. 2004;127:569-80.

36. Meirelles Lda S, Fontes AM, Covas DT, Caplan Al. Mechanisms involved in the therapeutic properties of mesenchymal stem cells. Cytokine Growth Factor Rev. 2009:20:419-27.

37. Lai D, Wang F, Dong Z, Zhang Q. Skin-derived mesenchymal stem cells help restore function to ovaries in a premature ovarian failure mouse model. PLoS One. 2014;9:e98749.

38. Liu T, Huang Y, Guo L, Cheng W, Zou G. CD44+/CD105+ human amniotic fluid mesenchymal stem cells survive and proliferate in the ovary long-term in a mouse model of chemotherapy-induced premature ovarian failure. Int $J$ Med Sci. 2012;9:592-602 
39. Tsagias N, Koliakos I, Karagiannis V, Eleftheriadou M, Koliakos GG. Isolation of mesenchymal stem cells using the total length of umbilical cord for transplantation purposes. Transfus Med. 2011;21:253-61.

40. Chong JJ, Chandrakanthan V, Xaymardan M, Asli NS, Li J, Ahmed I, Heffernan C, Menon MK, Scarlett CJ, Rashidianfar A, Biben C, Zoellner H, Colvin EK, Pimanda JE, Biankin AV, Zhou B, Pu WT, Prall OW, Harvey RP. Adult cardiac-resident MSC-like stem cells with a proepicardial origin. Cell Stem Cell. 2011;9:527-40.

41. Iorio R, Castellucci A, Ventriglia G, Teoli F, Cellini V, Macchiarelli G, Cecconi S. Ovarian toxicity: from environmental exposure to chemotherapy. Curr Pharm Des. 2014;20:5388-97.

42. Guo JQ, Gao X, Lin ZJ, Wu WZ, Huang LH, Dong HY, Chen J, Lu J, Fu YF, Wang J, Ma YJ, Chen XW, Wu ZX, He FQ, Yang SL, Liao LM, Zheng F, Tan JM. BMSCs reduce rat granulosa cell apoptosis induced by cisplatin and perimenopause. BMC Cell Biol. 2013;14:18.

43. Chen W, Xu X, Wang L, Bai G, Xiang W. Low expression of Mfn2 Is associated with mitochondrial damage and apoptosis of ovarian tissues in the premature ovarian failure model. PLoS One. 2015;10:e0136421.

44. Fox H. The pathology of premature ovarian failure. J Pathol. 1992;167:357-63.

45. Iwasaki M, Adachi Y, Minamino K, Suzuki Y, Zhang Y, Okigaki M, Nakano K, Koike Y, Wang J, Mukaide H, Taketani S, Mori Y, Takahashi H, Iwasaka T, Ikehara S. Mobilization of bone marrow cells by G-CSF rescues mice from cisplatin-induced renal failure, and M-CSF enhances the effects of G-CSF. J Am Soc Nephrol. 2005;16:658-66.

46. Zhou D, Tan RJ, Lin L, Zhou L, Liu Y. Activation of hepatocyte growth factor receptor, c-met, in renal tubules is required for renoprotection after acute kidney injury. Kidney Int. 2013;84:509-20.

47. Cheng K, Rai P, Plagov A, Lan X, Kumar D, Salhan D, Rehman S, Malhotra A, Bhargava K, Palestro CJ, Gupta S, Singhal PC. Transplantation of bone marrow-derived MSCs improves cisplatinum-induced renal injury through paracrine mechanisms. Exp Mol Pathol. 2013;94:466-73.

48. Fujita M, Ishihara M, Simizu M, Obara K, Ishizuka T, Saito Y, Yura H, Morimoto Y, Takase B, Matsui T, Kikuchi M, Maehara T. Vascularization in vivo caused by the controlled release of fibroblast growth factor-2 from an injectable chitosan/ non-anticoagulant heparin hydrogel. Biomaterials. 2004;25:699-706.

49. Ding L, Li X, Sun H, Su J, Lin N, Peault B, Song T, Yang J, Dai J, Hu Y. Transplantation of bone marrow mesenchymal stem cells on collagen scaffolds for the functional regeneration of injured rat uterus. Biomaterials. 2014;35:4888-900.

50. Wei $P$, Chen $X L$, Song $X X$, Han CS, Liu YX. VEGF, bFGF, and their receptors in the endometrium of rhesus monkey during menstrual cycle and early pregnancy. Mol Reprod Dev. 2004;68:456-62.

51. Jung Hwa Seo, Ji Hea Yu, Hwal Suh, Myung-Sun Kim, Sung-Rae Cho, Ken Arai, Jung Hwa Seo, Ji Hea Yu, Hwal Suh, Myung-Sun Kim, Sung-Rae Cho, Ken Arai. Fibroblast growth factor-2 induced by enriched environment enhances angiogenesis and motor function in chronic hypoxic-ischemic brain injury. PLOS ONE. 2013;8:e74405

52. Takehito Momose, Hirofumi Miyaji, Akihito Kato, Kosuke Ogawa, Takashi Yoshida, Erika Nishida, Syusuke Murakami, Yuta Kosen, Tsutomu Sugaya, Masamitsu Kawanami. Collagen Hydrogel Scaffold and Fibroblast Growth Factor-2 Accelerate Periodontal Healing of Class II Furcation Defects in Dog. Open Dent J. 2016;10:347-59.

\section{Submit your next manuscript to BioMed Central and we will help you at every step:}

- We accept pre-submission inquiries

- Our selector tool helps you to find the most relevant journal

- We provide round the clock customer support

- Convenient online submission

- Thorough peer review

- Inclusion in PubMed and all major indexing services

- Maximum visibility for your research

Submit your manuscript at www.biomedcentral.com/submit 